\title{
Editorial
}

\section{Achieving the promise of digital health information systems}

\author{
Gary L. Kreps \\ Center for Health and Risk Communication, George Mason University, Fairfax, VA, USA
}

\section{The failed promise of digital health information dissemination}

Many pundits, including me, proclaimed the advent of an era of digital health where health information systems would transform the delivery of health care and the promotion of public health. ${ }^{1-3}$ Digital health prophets described how health information systems would provide consumers and providers with relevant, timely, and influential health information to address challenging health issues and enhance health outcomes. However, the enthusiastic predictions about the amazing contributions of digital health programs for promoting public health has not reached fruition and the great promise of health information systems has resulted in limited returns. ${ }^{4}$ There is a long way to go for digital health information systems to reach their incredible potential.

\section{Digital media and the dissemination of relevant health information}

Timely, accurate, and appropriate health information is the most influential resource for guiding important health decisions. ${ }^{5}$ Consumers and providers depend on access to relevant health information to increase their understanding about complex health risks, problems, and interventions, to guide accurate diagnoses, to identify and respond to serious health risks, and to select the best health promotion interventions. Health care issues are often tremendously complex and revealing health information can reduce the many uncertainties consumers and providers confront in making important health decisions. Unfortunately, access to the best health information is often problematic. ${ }^{5}$ Due to numerous barriers to accessing health information, many people are seriously under-informed and misinformed about key health issues. ${ }^{6,7}$ Consumers are not the only ones who experience challenges in accessing health information. Even well trained health care professionals encounter problems accessing relevant health information needed for making important diagnostic and treatment decisions. Policy makers also struggle to access relevant information for guiding the design of effective health care regulations, programs, and practices. We need to design digital health information systems that do a better job of providing all participants in the modern health care system with the information they need to promote health!

\section{Factors that confound digital health}

There are a number of design issues that limit the effectiveness of digital health information systems. While many health websites provide a vast array of information about health conditions, treatments, research, providers, and facilities, the information is often provided in ways that is difficult for many people to access and understand. While, powerful search engines can provide online access to a broad range of relevant health websites, they often provide too much information about health issues that can be difficult for many information seekers to evaluate. Digital health decision support systems can interpret complex health problems and suggest best response strategies, but these systems are often difficult to use. Online support groups can be powerful forums for sharing relevant health information and support, yet many consumers who might benefit from these groups do not know how to access them. Numerous communication problems are preventing digital health information systems from achieving health promotion goals.

\section{Designing digital health information systems that communicate competently}

The best digital information systems must be designed to be interactive, personally relevant, relationally sensitive, involving, exciting, and easy to use. ${ }^{3,8-11}$ Regrettably, most e-health programs do not live up to these communication standards. They are typically overly complex, formal, technical, and difficult to use. The information they provide is often dispassionate, boring, and unimaginative. Even worse, the health promotion messages provided on many of these health information systems are insulting and disempowering to users. They provide overly directive and prescriptive statements that are typically presented in inflexible and static one-way messages, with minimal interaction and opportunities for involvement from consumers. Most current health information systems are often not much fun for users and it is not surprising these systems are not as well utilized or as effective as they might be!

There has been more emphasis on designing technically sophisticated health information systems than on making these system communicatively competent. While most current digital communication systems have been designed to store, process, and deliver vast amounts of health information, these systems often do not communicate health information in very meaningful, sensitive, and engaging ways to the diverse audiences who desperately need relevant health information. Health promoting communication is an intricate, interactive process that depends on the quality of relevant adaptive messages exchanged over time to inform important health decisions and influence health behaviours. ${ }^{5,10}$ Most digital health systems fail to fully engage users, leading to problems with information overload, and confusing rather than informing users, especially those users who experience health literacy challenges. Health literacy challenges are a pervasive and ubiquitous health communication impediment due to the complexity of making sense of many health problems, the bureaucracy of health care systems, the difficulties inherent in influencing health behaviours, and the physical, cognitive, and emotional challenges people typically face when they are feeling ill. When people are sick they may not think as clearly as usual, they are concerned, and perhaps 
fearful about their health conditions. They may be cognitively impaired by medications, pain, nausea, fatigue, and a whole host of conditions that keep them from exhibiting their best communication skills. It is not surprising that people who are confronting health problems often experience difficulties understanding complex health information.

\section{Strategies for enhancing digital communication systems}

When designing digital health information systems there are a number of key questions that should be asked. How engaging and interactive are these health information systems. Are these systems designed to capture audience attention? Do they communicate health information clearly and compellingly? Do they communicate humanely and sensitively? Do they adapt well to unique users? Do they promote immediacy? Immediacy is a powerful communication process that humanizes and intensifies interaction by promoting physical and emotional closeness, interpersonal comfort, engagement and caring, personal involvement, enthusiasm, authenticity, and enjoyment. ${ }^{8}$ How many digital health information systems achieve these humane communication goals? Not many! Research has shown that high levels of immediacy brings communicators closer together, enhances expressions of affect, increases cognitive and affective learning, increases perceptions of credibility and identification, enhances motivation and participation, encourages communication and feedback, and reduces resistance by promoting cooperation. ${ }^{8}$

The next generation of digital health information systems should be designed to demonstrate immediacy by communicating in friendly, animated, involving, exciting, comforting, and caring ways. Messages should be designed to be personal and familiar, involving, clear, and interesting to move users with relevant, interesting, and actionable health information. Strategic use of video, animation, dramatic narratives, virtual human agents, virtual reality, and interactive health games can promote immediacy. Immediate health information systems can enhance health promotion outcomes by increasing access (exposure) to relevant health information, improving the quality of health communication, increasing consumer involvement, and influencing both health behaviours and health outcomes. These systems can supplement traditional health care and health promotion activities as additional channels of health communication that can actively involve consumers in directing their own care, promote continuity of care, coordination of care, relieve demands on health care staff, reduce health care costs, increase the efficiency of health care services, and improve health outcomes. ${ }^{8}$

Communicatively competent digital health information systems can promote interactivity by providing opportunities for users to ask questions and receive meaningful answers. They will employ tailored message systems to provide specific and appropriate feedback. They will be designed using principles of artificial intelligence to relationally adapt over time to unique user responses and stored information about users gathered from electronic health records. ${ }^{8}$ These relationally sensitive, interactive, and adaptive digital health information systems will share control with users by encouraging user input and direction based on individual user needs and preferences. They will also provide congruent, empathetic, and personally sensitive messages that demonstrate concern and respect for users. A new generation of communicatively competent health information systems can achieve the tremendous promise of digital health for enhancing health outcomes.

Correspondence: Gary L. Kreps, Department of Communication, Center for Health and Risk Communication, George Mason University, 4400 University Drive, MS 3D6, Fairfax, VA 22030, USA.

Tel.: +1.703.993.1094 - Fax: +1.703. 993.1096.

E-mail: gkreps@gmu.edu

Received for publication: 1 December 2014.

Accepted for publication: 1 December 2014.

(C) Copyright G.L. Kreps, 2014

Licensee PAGEPress, Italy

Journal of Public Health Research 2014; 3:471

doi:10.4081/jphr.2014.471

This work is licensed under a Creative Commons Attribution NonCommercial 3.0 License (CC BY-NC 3.0).

\section{References}

1. Kreps GL. Health communication and information technology. The Electronic Journal of Communication / La Revue Electronique de Communication/La Reve Electronique de Communication. 2002. Available from: http://www.cios.org/www/ejc/v11n3.htm\# Intro2

2. Kreps GL. E-health: technology-mediated health communication. J Health Psychol 2003;8:5-6.

3. Neuhauser L, Kreps GL. The advent of e-health: how interactive media are transforming health communication. Medien Kommunikationswissenschaft 2003;51:541-56.

4. Kreps GL, Neuhauser L. New directions in ehealth communication: opportunities and challenges. Patient Educ Couns 2010;78:329-336.

5. Kreps GL. The pervasive role of information in health and health care: implications for health communication policy. In: Anderson J, Ed. Communication yearbook 11. Newbury Park, CA: Sage; 1988. pp 238-276.

6. Kreps GL. Consumer control over and access to health information. Ann Fam Med 2012;10:428-34.

7. Kreps GL. The information revolution and the changing face of health communication in modern society. J Health Psychol 2011; 16:192-3.

8. Kreps GL, Neuhauser L. Artificial intelligence and immediacy: designing health communication to personally engage consumers and providers. Patient Educ Couns 2013;92: 205-10.

9. Kreps GL, Neuhauser L. E-health and health promotion. J Comput Mediat Commun 2010;15:527-9.

10. Neuhauser L, Kreps GL. Ehealth communication and behavior change: promise and performance. Soc Semiot 2010;20:7-24.

11. Neuhauser L, Kreps GL. Rethinking communication in the e-health era. J Health Psychol 2003;8:7-22. 